\title{
Endoscopic Sleeve Gastroplasty - A New Tool to Manage Obesity
}

\author{
Deepanshu Jain ${ }^{1}$, Bharat Singh Bhandari ${ }^{2}$, Ankit Arora $^{2}$ and Shashideep Singhal ${ }^{2}$ \\ Division of Gastroenterology, ${ }^{1}$ Department of Internal Medicine, Albert Einstein Medical Center, Philadelphia, PA, ${ }^{2} \mathrm{Hepatology}$ and Nutrition, \\ University of Texas Health Science Center at Houston, Houston, TX, USA
}

\begin{abstract}
Obesity is a growing pandemic across the world. Dietary restrictions and behavior modifications alone have a limited benefit. Bariatric surgery, despite being the current gold standard, has limited acceptance by patients due to cost and associated morbidity. In our review, we have discussed nine original studies describing endoscopic sleeve gastroplasty (ESG). A total of 172 subjects successfully underwent ESG. Of 65 subjects with follow up data, 95.4\% (62/65) had intact gastric sleeve confirmed via esophagogastroduodenoscopy or oral contrast study at the end of study specific follow up interval (the longest being 6 months). Individual studies reported a technical success rate for intact gastric sleeve from as low as $50 \%$ to as high as $100 \%$. A statistically significant $(p<0.05)$ weight loss was reported in seven of the eight studies with available data. None of the patients experienced any intra-procedure complications, and approximately $2.3 \%$ (4/172) of patients experienced major post-procedure complications; however, no mortality was reported. Majority of the studies reported relatively high incidence of minor post-procedure complications, which improved with symptomatic treatment alone. Good patient tolerance with comparable clinical efficacy in achieving and sustaining desired weight loss makes ESG an attractive option to consider among other bariatric therapies. Clin Endosc 2017;50:552-561
\end{abstract}

Key Words: Endoscopic sleeve gastroplasty; Obesity; Endoscopic bariatric therapy; Body mass index; Extra weight loss

\section{INTRODUCTION}

Obesity is a growing pandemic across the world. The Centre for Disease Control and Prevention (CDC) defines obesity as a body mass index (BMI) of $30 \mathrm{~kg} / \mathrm{m}^{2}$ or higher. Due to differences in body fat distribution and associated health risks, obesity for Asians is defined as a BMI of $25 \mathrm{~kg} /$ $\mathrm{m}^{2}$ or higher. According to The National Health and Nutrition Examination Survey (NHANES) data, obesity rates in adults from the USA have increased from $32.9 \%$ in $2003-2004$ to $38 \%$ in 2013-2014. ${ }^{1}$ As of September 2016, adult obesity rates are $>35 \%$ in four states, $30 \%$ in 25 states, and $>20 \%$ in all states

Received: February 20, 2017 Revised: April 13, 2017

Accepted: April 26, 2017

Correspondence: Shashideep Singhal

Division of Gastroenterology, Hepatology and Nutrition, University of Texas Health Science Center at Houston, 6431 Fannin Street, MSB 4.234, Houston, TX 77030, USA

Tel: +1-713-500-6677, Fax: +1-713-500-6699, E-mail: sdsinghal@gmail.com

(cc) This is an Open Access article distributed under the terms of the Creative Commons Attribution Non-Commercial License (http://creativecommons.org/ licenses/by-nc/3.0) which permits unrestricted non-commercial use, distribution, and reproduction in any medium, provided the original work is properly cited. of the USA. ${ }^{2}$ Obesity is associated with increased risk of death. Adams et al. studied the risk of death in a large prospective cohort of over 500,000 men and women aged 50 to 71 years, and reported that the risk of death was increased by $20 \%$ to $40 \%$ among overweight individuals and by two to at least three times among obese persons who had never smoked. ${ }^{3}$ Obesity is associated with a number of diseases, including hypertension, heart disease, diabetes, and stroke. Achieving and maintaining desired weight loss by means of dietary restrictions and behavior modifications alone has remained nonproductive. Chang et al. did a systematic review and meta-analysis of 164 studies, analyzing over 160,000 patients who underwent bariatric surgery, and concluded that bariatric surgery provides substantial and sustained effects on weight loss and ameliorates obesity-attributable comorbidities in the majority of bariatric patients, although risks of complication, reoperation, and death exist. ${ }^{4}$ However, due to a burden of cost and resources, it is not feasible to provide surgery to all those who may benefit from it. Worldwide estimates suggest that only $<1 \%$ of the target population has access to surgery. ${ }^{5}$ Endoscopic bariatric therapies are minimally invasive techniques targeted to achieve comparable results to current gold 
standard treatment (bariatric surgery) for obesity but at a more affordable cost and a lower complication rate. It includes endobarrier devices like duodenojejunal bypass sleeve and gastroduodenojejunal bypass sleeve, endoscopic placement of space occupying devices like intra-gastric balloons and endoscopic placement of full thickness sutures in endoscopic sleeve gastroplasty (ESG), and Rouxen-Y Gastric bypass revision. ${ }^{6,7}$

In this review, we have summarized individual single center prospective studies describing the ESG technique as a treatment modality for obese subjects.

\section{MATERIALS AND METHODS}

Two authors individually reviewed English literature from inception through December 2016. PubMed and Google scholar were used to identify peer reviewed original and review articles using the following key words: endoscopic sleeve gastroplasty, obesity, endoscopic bariatric therapy. Only studies in humans were selected. The references of pertinent studies were manually searched to identify additional relevant studies. Search results yielded nine single center studies. ${ }^{8-14}$ We included all nine studies in the review since it was not clear if the previous studies by the same author were excluded in their respective new publications. To make conclusions we only used studies with the longest patient enrollment time period from each institute to avoid any confounding effect from published duplicate data. The indications, procedural details, technical success rate, clinical outcomes, complications, and limitations were reviewed for each study.

\section{RESULTS}

We selected nine original studies to be included in this review article. Four studies were from the USA, which included one single center feasibility study ${ }^{8}$ and three single center prospective studies. ${ }^{9-11}$ Four studies were from Spain, all of which were single center prospective studies. ${ }^{12-15}$ One case report from Brazil was also included. ${ }^{16}$ All the studies have been summarized in Table 1. Technical success was determined in terms of intact endoscopic sleeve post-procedure evaluated using oral contrast studies and endoscopy at varied intervals. Clinical success was evaluated using weight reduction parameters such as excess weight loss (EWL) percentage, BMI, waist circumference, and weight measurements at intervals. One study reported effect of ESG on parameters other than weight

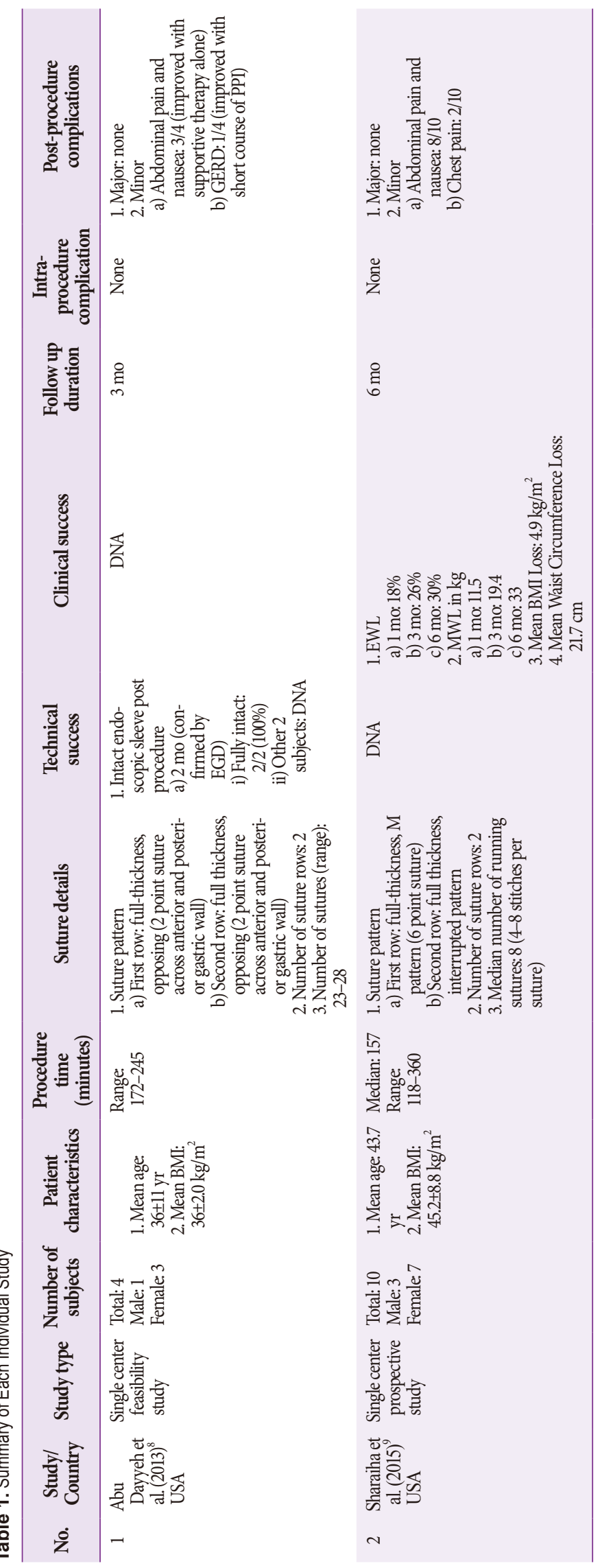




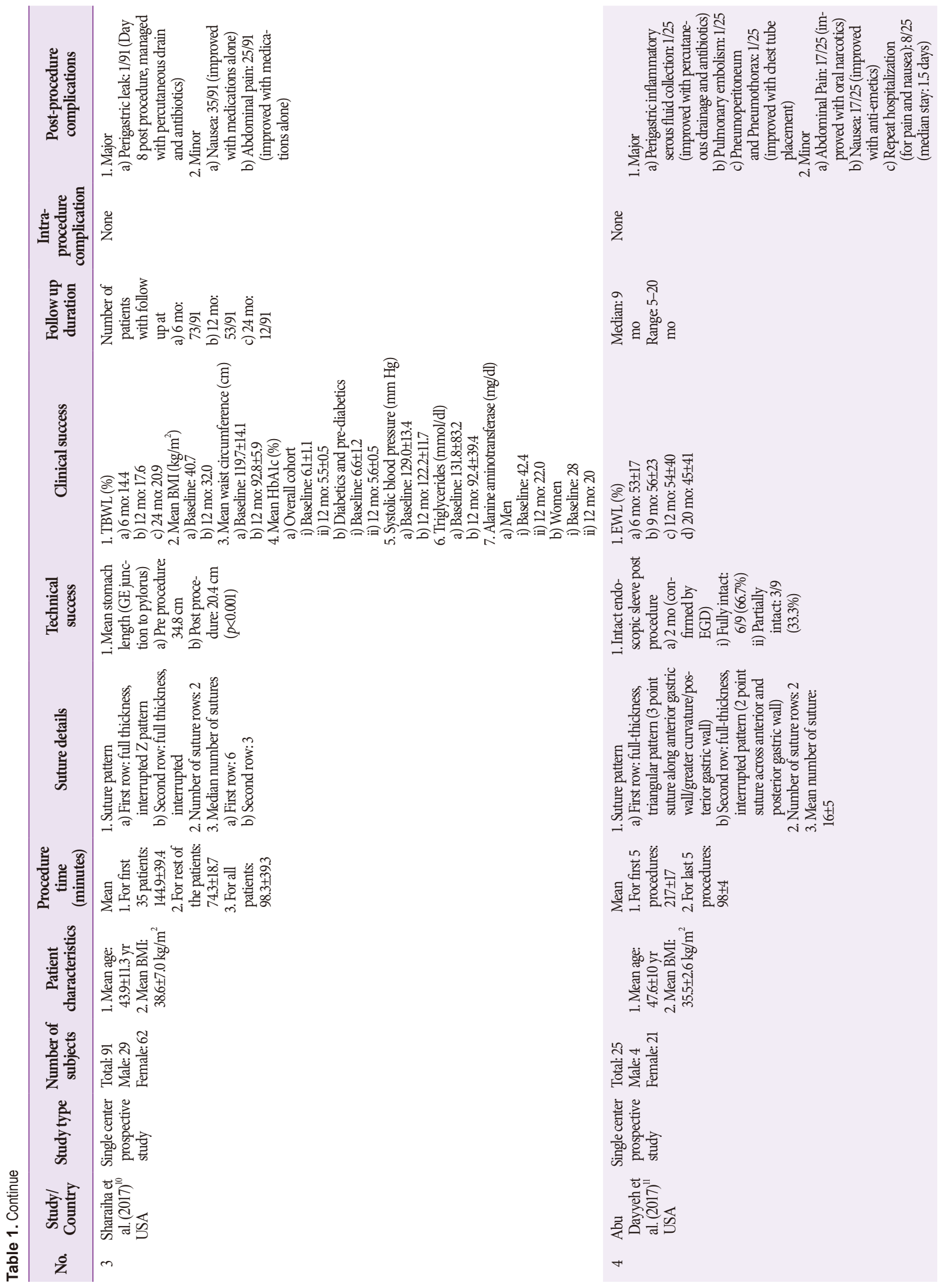




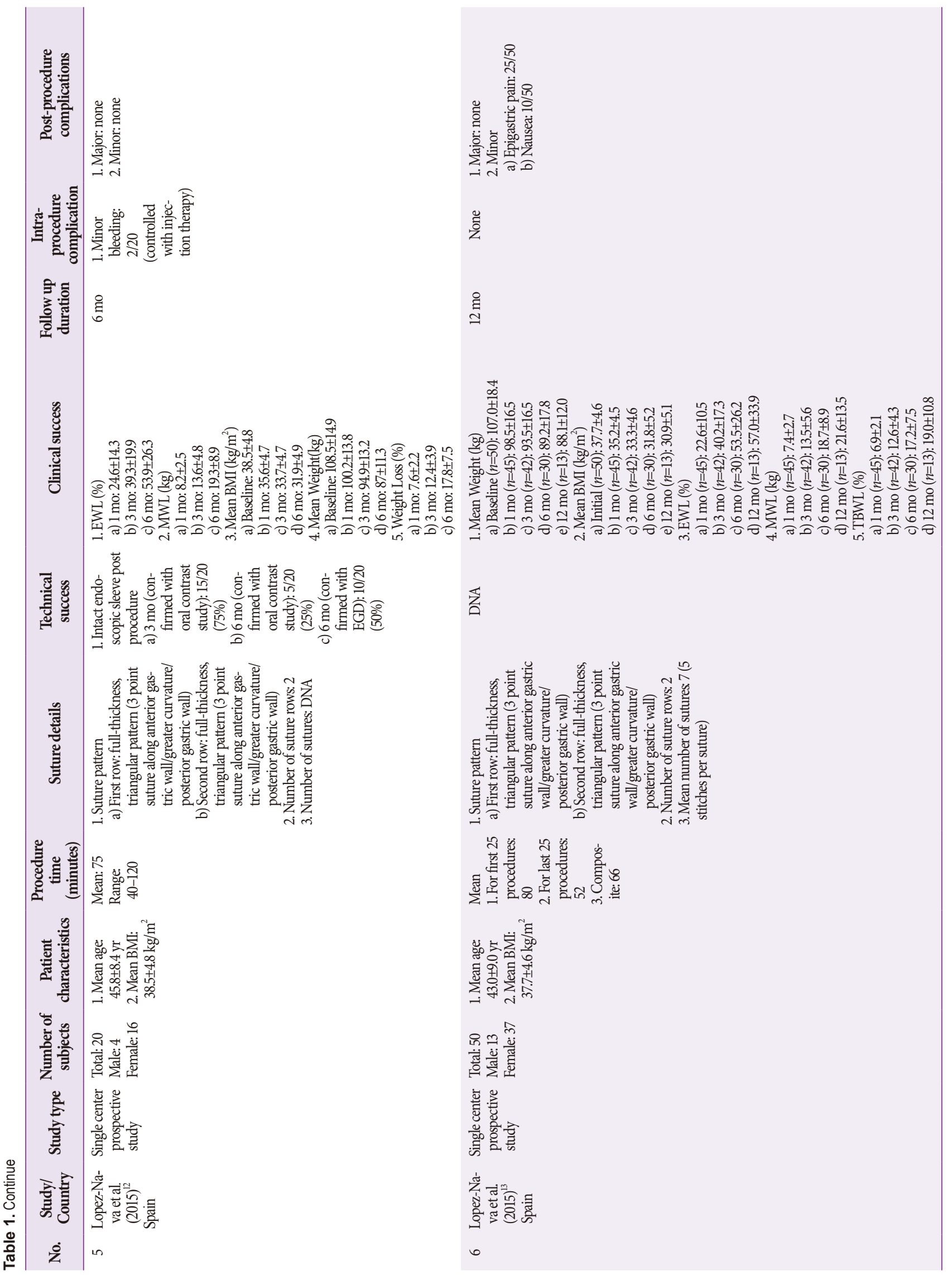




\section{$C_{\text {clincal endoscopy }}$}

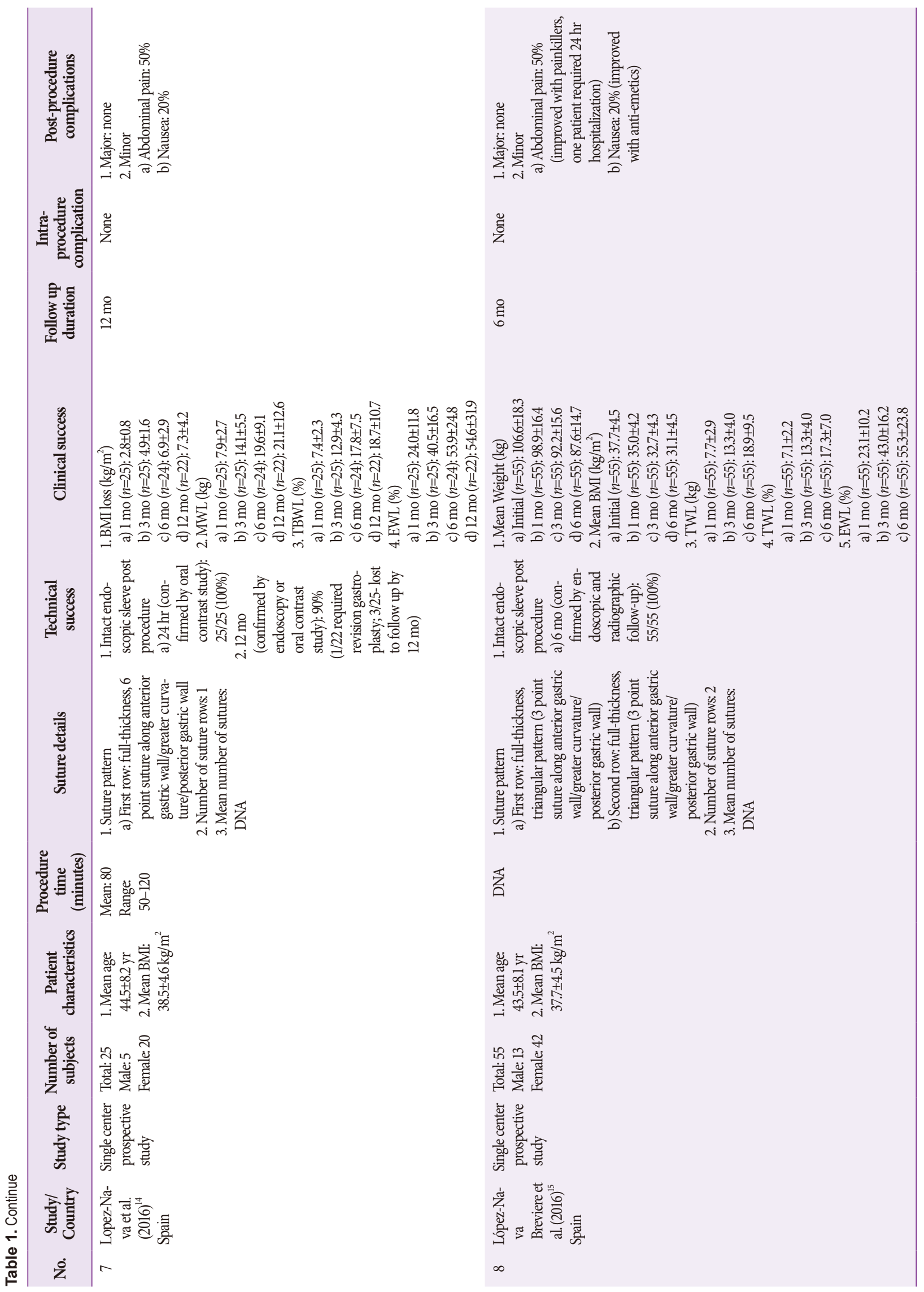




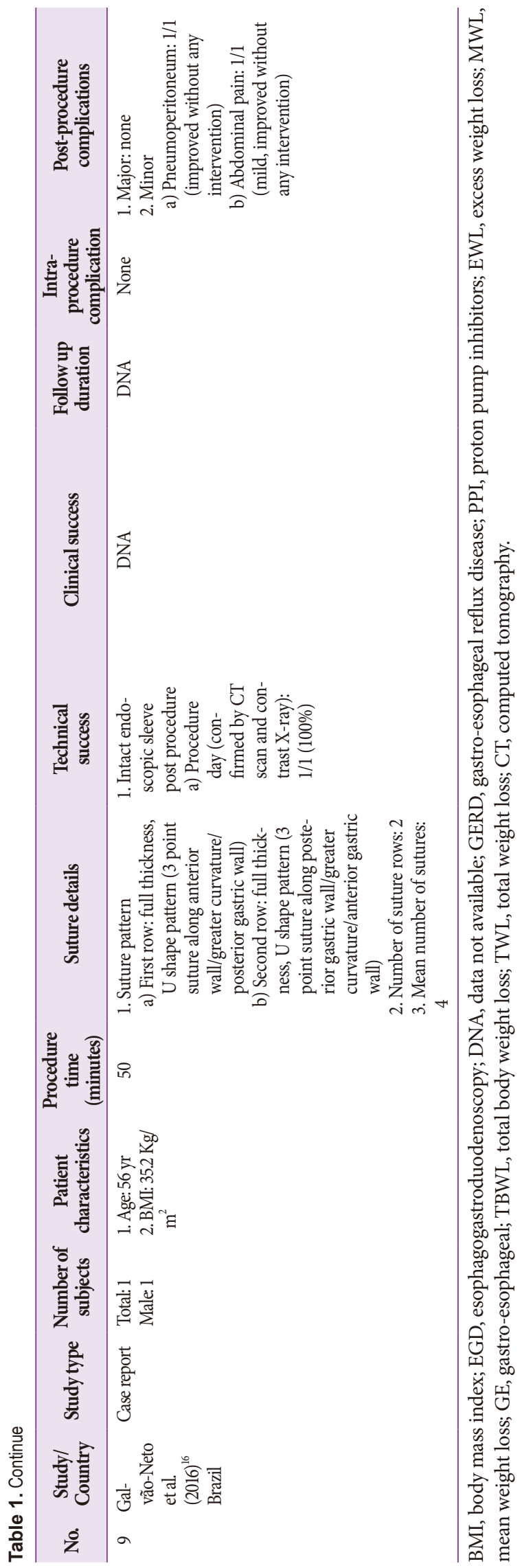

loss, including change in blood pressure, triglyceride level, diabetes control, and liver function tests. ${ }^{10}$ Intra-procedure and post-procedure complications were also evaluated to determine safety of the procedure, which mainly included bleeding, post-operative abdominal pain, nausea, and vomiting.

\section{DISCUSSION}

\section{Patient characteristics}

The mean age of patients who had failed to lose weight with non-surgical methods across studies ranged from 36 to 56 years and mean BMI ranged from 35.2 to $45.2 \mathrm{~kg} / \mathrm{m}^{2}$. 816

\section{Contraindications}

Some common contraindications considered by authors before considering the subjects for ESG included presence of neoplastic lesions, ${ }^{10,12-15}$ potential bleeding gastric lesions (ulcers, gastritis) ${ }_{10-15}^{15}$ coagulopathy, ${ }^{10-15}$ hiatal hernia, ${ }^{11,15}$ psychiatric disorders, ${ }^{10,12-15}$ pregnancy, ${ }^{11}$ significant medical comorbidities precluding sedation, ${ }^{10}$ hiatus hernia $>3 \mathrm{~cm},{ }^{15}$ or history of prior gastric surgery. ${ }^{11,14}$

\section{Anesthesia}

All the authors have reported the use of general anesthesia (GA) to perform their respective procedures. ${ }^{8-16}$ Carbon dioxide $\left(\mathrm{CO}_{2}\right)$ insufflation was used to distend the gastric lumen. ${ }^{8-16}$

\section{Procedure time}

The procedure time varied widely across different studies from a minimum of 40 minutes $^{12}$ to a maximum of 360 minutes. ${ }^{9}$ Abu Dayyeh et al. reported the mean procedure time for the first five procedures as $217 \pm 17$ minutes and for the last five patients as $98 \pm 4$ minutes. ${ }^{11}$ Lopez-Nava et al. reported the mean procedure time for the first 25 patients as 80 minutes and for the last 25 patients as 52 minutes. ${ }^{13}$ Similarly, Sharaiha et al. reported a mean procedure time of $144.9 \pm 39.4$ minutes for the first 35 patients, which reduced to $74.3 \pm 18.7 \mathrm{~min}$ in the remaining 56 patients. ${ }^{10}$ These trends are suggestive of a learning curve of endoscopist performing the procedure leading to a progressive decrease in procedure time over the course of study.

\section{Technique and sutures}

Most authors used the endoscopic suturing device (OverStitch; Apollo Endosurgery, Inc., Austin, TX, USA) to place sutures endoluminally. The technique and suture pattern varied across the studies. ${ }^{8-16}$ Abu Dayyeh et al. used a series of endoluminally placed full-thickness closely spaced interrupted 
sutures (two point suture across anterior and posterior gastric wall) from the prepyloric antrum to the gastroesophageal junction forming two rows of sutures. ${ }^{8}$ Each subject required 23-28 sutures. ${ }^{8}$ In their second study, author used a slightly different technique, where the endoscopic gastric sleeve was created by using two rows of sutures using a different pattern. The first row was formed using a triangular pattern (three point suture across the greater curvature, anterior and posterior gastric wall) for creation of a narrow sleeve and the second row of sutures was placed over the length of the central sleeve in an interrupted pattern (two point suture across the anterior and posterior gastric wall) to further reduce gastric volume and reinforce the sleeve. ${ }^{11}$ Author used an average of $16 \pm 5$ sutures per subject in his study. ${ }^{11}$ Sharaiha et al. reduced gastric volume by endoscopic placement of two rows of full-thickness sutures via endoscopic suturing device. ${ }^{9}$ The first suture row was created in M pattern (six point sutures) and the second suture row was created with an interrupted pattern. ${ }^{9}$ Four to eight stiches were used to create each suture and author used a median number of eight sutures per subject. ${ }^{9}$ In their second study, the gastric sleeve was created by two rows of full thickness interrupted sutures. ${ }^{10}$ The first row was created in a Z pattern requiring a median number of six sutures, whereas the second row was created by using a median number of three sutures. ${ }^{10}$ In three studies by Lopez-Nava et al,, author used the endoscopic suturing device to place two rows of full thickness sutures, each in a triangular pattern (three point suture across the greater curvature, anterior and posterior gastric wall) to create the gastric sleeve. ${ }^{12,13,15}$ In another study, Lopez-Nava et al. created a single row of interrupted sutures from distal to proximal body. ${ }^{14}$ Each suture consisted of six bites along the anterior gastric wall, greater curvature, and the posterior gastric wall. ${ }^{14}$ In the case report, author created gastric sleeve by two rows of full thickness $U$ shaped sutures (three point suture across the anterior gastric wall, greater curvature, and posterior wall). ${ }^{16}$ Mean number of four sutures were used for each subject. ${ }^{16}$

\section{Physiological analysis}

In one of the study by Abu Dayyeh et al., author did a pilot sub-study on four subjects to assess metabolic and physiological alterations that occur in response to ESG. ${ }^{11}$ They reported a 90 minute increase in gastric emptying time for $50 \%$ of solids at 3 months post-ESG ( $p=0.03$ ), but no significant change for liquids. At 3 months post-ESG, the time to reach maximum fullness on meal tolerance test decreased to $11.5 \pm 2.3 \mathrm{~min}$ as compared with $35.2 \pm 9.9$ min before ESG ( $p=0.01)$. This was likely a result of $59 \%$ decrease in the caloric intake required to reach satiety ( $p=0.003$ ). Despite weight loss, there was a decrease of $29.4 \%$ in active fasting and postprandial ghrelin levels at 3 months post ESG ( $p=0.1$ ). No significant change was noted in leptin, glucagon-like peptide-1, and peptide YY levels. Based on a fasting homeostatic model, a decrease in insulin resistance score $(p=0.06)$, area under the curve for post prandial glucose $(p=0.005)$, and insulin $(p=0.17)$ was seen at 3 months post-ESG.

\section{Outcome}

\section{Technical success}

The technical success rate was calculated using post-procedure gastric sleeve and suture intactness. The sleeve and sutures were evaluated using endoscopy and oral contrast studies done post-procedure at various study specific intervals. Abu Dayyeh et al. reported 100\% (2/2) technical success rate at 2 months interval in their first study. In their second study, 66.7\% (6/9) patients had fully intact gastric sleeve and $33.3 \%(3 / 9)$ had partially intact sleeve at 2 months of follow up. ${ }^{11}$ Lopez-Nava et al. did oral contrast studies in 20 patients and found that $75 \%(15 / 20)$ and 25\% (5/20) had intact sleeve at 3 and 6 months post-procedure, respectively. ${ }^{12}$ They also performed an endoscopic evaluation at 6 months in all patients and found $50 \%$ technical success rate. ${ }^{12}$ Interestingly, the technical success rate derived from endoscopic visualization was twice as high as the number derived based on the oral contrast study. Economically, endoscopic follow up may not be a feasible option for all subjects in routine examination but interpretation of oral contrast studies in this scenario needs re-consideration. In the other study, Lopez-Nava et al. had technical success in $100 \%(25 / 25)$ and $90 \%$ of subjects at 24 hours and 12 months post-procedure, respectively, based on oral contrast studies. ${ }^{14}$ Of 22 patients who were still followed up at one year, one subject required revision gastroplasty. ${ }^{14}$ López-Nava Breviere et al. reported 100\% intact gastric sleeves in all 55 patients at 6 months of post-procedure as confirmed by both endoscopic and radiologic tests. ${ }^{15}$ Galvão-Neto et al. reported intact gastric sleeve in his case report just hours post-procedure based on a computed tomography scan and contrast X-ray. ${ }^{16}$

Sharaiha et al. reported a decrease in mean gastric length (gastro-esophageal junction to pylorus) from $34.8 \mathrm{~cm}$ (preESG) to $20.4 \mathrm{~cm}$ (post-ESG; $p<0.001)^{10}$

\section{Clinical success}

Authors have used different parameters to illustrate the efficacy of gastroplasty in achieving weight reduction. Clinical success was measured in terms of direct parameters, such as mean weight, mean BMI, mean weight loss (MWL), average percentage of EWL, and total body weight loss (TBWL) percentage. Sharaiha et al. in their prospective study reported a 
MWL and EWL of $11.5 \mathrm{~kg}$ and $18 \%$ at 1 month, $19.4 \mathrm{~kg}$ and $26 \%$ at 3 months, and $33.0 \mathrm{~kg}$ and $30 \%$ at 6 months, respectively. ${ }^{9}$ At 6 months of follow up, there was a mean BMI loss of $4.9 \mathrm{~kg} / \mathrm{m}^{2}(p=0.0004)$ and a mean waist circumference loss of $21.7 \mathrm{~cm}(p=0.003))^{9}$ Similarly, in another study by the same author, a significant decrement was observed in a mean BMI and mean waist circumference at 12 months $(p<0.001) .{ }^{10}$ TBWL at 6,12 , and 24 months was $14.4 \%, 17.6 \%$, and $20.9 \%$, respectively $(p<0.001) .{ }^{10}$ In a study on 25 patients (of whom only eight patients underwent 20 months follow up), Abu Dayyeh et al. reported EWL of $53 \% \pm 17 \%$, $56 \% \pm 23 \%, 54 \% \pm 40 \%$, and $45 \% \pm 41 \%(p<0.1)$ at $6,9,12$, and 20 months, respectively. ${ }^{11}$ Five of the eight patients $(62.5 \%)$ with 20 months follow up had an excellent durable response with an EWL of $72 \% \pm 21.8 \%$, in contrast to the remaining three subjects who regained all the lost weight. ${ }^{11}$ Lopez-Nava et al. reported a statistically significant weight reduction from $108.5 \pm 14.9 \mathrm{~kg}$ to $100.2 \pm 13.8,94.9 \pm 13.2,87 \pm 11.3 \mathrm{~kg}$ at 1,3 , and 6 months follow up $(p<0.05) .^{12}$ A similar trend was observed in BMI over time. ${ }^{12}$ In addition, a progressive increment in EWL (24.6 \pm 14.3 at 1 month, $39.3 \pm 19.9$ at 3 months, $53.9 \pm 26.3$ at 6 months) and percentage weight loss (7.6 \pm 2.2 at 1 month, $12.4 \pm 3.9$ at 3 months, $17.8 \pm 7.5$ at 6 months) was observed. ${ }^{12}$ In another study, Lopez-Nava et al. studied 50 subjects and reported reduction in mean weight from $107.0 \pm 18.4 \mathrm{~kg}$ (baseline) to $98.5 \pm 16.5,93.5 \pm 16.5,89.2 \pm 17.8$, and $88.1 \pm 12.0 \mathrm{~kg}$ at one, three, six, and 12 months follow up $(p<0.05){ }^{13}$ The mean percentage TBWL and EWL was $19.0 \pm 10.8$ and $57.0 \pm 33.9$ at one year follow-up (13 patients), respectively. ${ }^{13}$ Another study by the same author included 25 patients and followed them up for 12 months post-ESG. ${ }^{14}$ There was a significant difference in all weight-based parameters over time $(p<0.05) .{ }^{13}$ At 12 month follow up of 22 subjects, the mean BMI loss, MWL, TBWL, and EWL was $7.3 \pm 4.2 \mathrm{~kg}, 21.1 \pm 12.6 \mathrm{~kg}, 18.7 \% \pm 10.7 \%$, and $54.6 \% \pm 31.9 \%$, respectively. ${ }^{14}$ In this study, the greatest decrease in weight was seen in the first month post-ESG when all subjects were restricted from solid food. ${ }^{14}$ Individuals with a higher number of nutritional and psychological contacts had more weight loss $(p<0.05){ }^{14}$ López-Nava Breviere et al. performed ESG in 55 patients and followed them up for a period of 6 months. ${ }^{15} \mathrm{~A}$ statistically significant decrease in mean weight (106.6 $\pm 18.3 \mathrm{~kg}$ before ESG, $98.9 \pm 16.4 \mathrm{~kg}$ at 1 month, $92.2 \pm 15.6 \mathrm{~kg}$ at 3 months, and $87.6 \pm 14.7 \mathrm{~kg}$ at 6 months) and BMI $\left(37.7 \pm 4.5 \mathrm{~kg} / \mathrm{m}^{2}\right.$ before ESG, $35.0 \pm 4.2 \mathrm{~kg} / \mathrm{m}^{2}$ at 1 month, $32.7 \pm 4.3 \mathrm{~kg} / \mathrm{m}^{2}$ at 3 months, and $31.1 \pm 4.5 \mathrm{~kg} / \mathrm{m}^{2}$ at 6 months) was reported $(p<0.05) .{ }^{15}$ The percentage decrease in EWL (23.1 \pm 10.2 at 1 month, $43.0 \pm 16.2$ at 3 months, and $55.3 \pm 23.8$ at 6 months) and total weight loss (TWL) (7.1 \pm 2.2 at 1 month, $13.3 \pm 4.0$ at 3 months, and $17.3 \pm 7.0$ at 6 months) was also significant $(p<0.05){ }^{15}$ Statistically, results were significant in majority of the studies for weight loss, suggesting ESG to be an effective modality to treat obesity.

A prospective study by Sharaiha et al. is the first study of its type to report effect of ESG on clinical parameters other than body weight. ${ }^{10}$ Post-ESG, at 12 months follow up, a decrease in mean HbAlc (6.1\% $\pm 1.1 \%$ to $5.5 \% \pm 0.5 \%$; $p=0.05)$, mean systolic blood pressure $(129.0 \pm 13.4 \mathrm{~mm} \mathrm{Hg}$ to $122.2 \pm 11.7 \mathrm{~mm} \mathrm{Hg}$; $p=0.023)$, mean triglycerides $(131.8 \pm 83.2 \mathrm{mmol} / \mathrm{dl}$ to $92.4 \pm 39.4$ $\mathrm{mmol} / \mathrm{dl} ; p=0.017$ ), and mean alanine aminotransferase (ALT) $(32.3 \pm 16.4 \mathrm{mg} / \mathrm{dl}$ to $20.7 \pm 11.4 \mathrm{mg} / \mathrm{dl} ; p<0.001)$ was observed. ${ }^{10}$ Obesity is a known risk factor for diabetes, hypertension, hypertriglyceridemia, and non-alcoholic fatty liver disease. ESG mediated weight loss among obese subjects has potential to improve clinical outcome for associated medical conditions.

\section{Post-procedure diet}

Post-procedure dietary restrictions were advised to patients in all the studies. Abu Dayyeh et al. used post-procedural translational diet consisting of 4 weeks of liquid protein shakes and 2 weeks of pureed diet before transitioning to a regular diet in their prospective studies. ${ }^{8,11}$ Sharaiha et al. followed a post-procedural translational diet consisting of 2 weeks of liquid protein shakes and 2 weeks of pureed diet before they transitioned to a regular diet. ${ }^{9,10}$ The post-procedural diet was designed to provide $70 \mathrm{~g}$ of protein and 1,000-1,200 calories per day. ${ }^{9,10}$ In addition, subjects were encouraged to drink 56 ounces of non-caloric fluids per day. ${ }^{9,10}$ Lopez-Nava et al. followed a similar diet plan in all three of their studies. ${ }^{12-14}$ A liquid diet was started on the day before the procedure, which continued for at least 2 weeks after the procedure. The diet progressed from hypocaloric liquids to small semisolid meals over 4 weeks. An exercise plan that avoids an increase in intra-abdominal pressure was recommended during the first month. Initially, walking was encouraged together with a progressive increase in the intensity of exercise as the diet progressed. ${ }^{12-14}$ López-Nava Breviere et al. followed a progressive consistency liquid diet during the first month post-ESG. ${ }^{15}$ Similarly, a residue free liquid diet was fed to the subjects post-ESG. ${ }^{16}$

\section{Adverse events}

\section{Intraoperative complications}

None of the authors has reported any major adverse events at the time of the procedure. ${ }^{8-16}$ Lopez-Nava et al. noticed minor bleeding in $10 \%(2 / 20)$ of subjects during the procedure, which was successfully controlled with injection therapy. ${ }^{12}$

\section{Minor post-procedure complications}

The authors have reported a wide spectrum of non-serious 
post-procedure adverse events. These include nausea, ${ }^{8-11,13-15}$ abdominal pain, ${ }^{8-11,14-16}$ chest/epigastric pain, ${ }^{8,913}$ asymptomatic pneumoperitoneum, ${ }^{16}$ and gastro-esophageal reflux. ${ }^{8}$ All of these adverse events were managed conservatively using painkillers, oral narcotics, proton pump inhibitors, antiemetic medications, or just observation. In the study by Abu Dayyeh et al., eight out of 25 patients (32\%) required repeated hospitalization for pain and nausea with a median stay of 1.5 days. ${ }^{11}$ Similarly, one out of 55 patients (1.8\%) required hospitalization for abdominal pain that improved with painkillers alone within 24 hours. ${ }^{15}$

Overall, all studies ${ }^{8-11,13-16}$ except one ${ }^{12}$ reported a relatively high prevalence of minor adverse events post-ESG that were successfully controlled with conservative management alone. $^{8-16}$

\section{Major post-procedure complications}

There were no major post-procedure complications in most of the studies ${ }^{8,9,12-16}$ except two. ${ }^{10,11}$ Sharaiha et al. reported one subject who developed perigastric leak on day eight postESG. ${ }^{10}$ Subject was treated with percutaneous drain and antibiotics with good recovery. ${ }^{10}$ Abu Dayyeh et al. reported three serious adverse events in their study. ${ }^{11}$ These included perigastric inflammatory serous fluid collection (1/25), pulmonary embolism (1/25), and pneumoperitoneum with pneumotho$\operatorname{rax}(1 / 25) .{ }^{11}$ Perigastric inflammatory serous fluid collection improved with percutaneous drainage and antibiotics, while pneumothorax improved with chest tube placement. All of these patients recovered fully with no need for any surgical intervention. Authors changed their clinical protocol midway after these major complications. To decrease the risk of pulmonary embolism, intermittent pneumatic compression devices were placed on patients' lower extremities during the procedure and a dose of prophylactic subcutaneous heparin was administered during the endoscopic procedure. To decrease a risk of pneumoperitoneum, $\mathrm{CO}_{2}$ insufflation was minimized during suture placement and the abdomen was closely monitored for distention during the procedure. Lastly, no more attempts were made to reduce the fundus because posterior aspect of the gastric fundus was considered the most vulnerable location for a post-procedure leak secondary to its thin wall and tension created by sutures at this location that approximate the fundus anteriorly to the gastroesophageal junction. Since the adoption of these changes, no more complications were reported in the remaining study period. ${ }^{11}$

ESG is an effective but a relatively new technique as a treatment option for obese patients. Currently, the procedure is being performed only at highly specialized centers. A high risk of complications (mostly minor) limits its universal applicability. Evolving experience is expected to lead to refinement of current technique and lowering of complication rate.

\section{CONCLUSIONS}

ESG, a new endoscopic bariatric technique, creates structural and physiologic changes to achieve and maintain desired weight loss. Five ${ }^{8,9,12-14}$ out of nine studies, ${ }^{8-16}$ as discussed above, were from same institutes with overlapping time periods for subject enrollment. To avoid any confounding effect from duplicate published data, only studies with longest enrollment time from the respective institutes were included for calculation of composite success and complication rates. ${ }^{10,11,15,16}$ A total of 172 subjects in four individual studies successfully underwent ESG. ${ }^{10,11,15,16}$ Of the 65 subjects with follow up data, 95.4\% (62/65) had intact gastric sleeve either confirmed via esophagogastroduodenoscopy or oral contrast study at the end of study-specific follow up interval. ${ }^{10,11,15,16}$ Individually, studies reported a technical success rate for intact gastric sleeve from as low as $50 \%$ to as high as $100 \%{ }^{8-16}$ A statistically significant $(p<0.05)$ weight loss was reported in seven out of eight studies with available data. ${ }^{8-15}$ None of the patients experienced any intra-procedure complications $^{10,11,15,16}$ and approximately, 2.3\% (4/172) of patients experienced major post-procedure complication; however, no mortality was reported. ${ }^{10,11,15,16}$ Majority of the studies reported relatively high incidence for minor post-procedure complications, which improved with symptomatic treatment alone. A decrease in complication rate and total procedure time was noted with the progress of individual studies suggestive of a learning curve associated with the technique. So far, experience with ESG is small but promising. Low complication rate with comparable clinical efficacy in achieving and sustaining desired weight loss makes it an attractive option to consider among other endoscopic bariatric therapies.

\section{Conflicts of Interest}

The authors have no financial conflicts of interest.

\section{Author Contribution}

Conceptualization: Deepanshu Jain, Shashideep Singhal

Data curation: DJ, Bharat Singh Bhandari, Ankit Arora

Formal analysis: DJ

Methodology: DJ

Supervision: SS

Writing-original draft: DJ, BSB

Writing-review\&editing: SS

\section{REFERENCES}

1. Flegal KM, Kruszon-Moran D, Carroll MD, Fryar CD, Ogden CL. Trends in obesity among adults in the United States, 2005 to 2014 
JAMA 2016;315:2284-2291.

2. Trust for Americas Health. The state of obesity 2016 [Internet]. Washington, DC: Trust for America’s Health; c2016 [updated 2016 Sep; cited 2016 Nov 5]. Available from: http://stateofobesity.org/files/stateofobesity2016.pdf.

3. Adams KF, Schatzkin A, Harris TB, et al. Overweight, obesity, and mortality in a large prospective cohort of persons 50 to 71 years old. N Engl J Med 2006;355:763-778.

4. Chang SH, Stoll CR, Song J, Varela JE, Eagon CJ, Colditz GA. The effectiveness and risks of bariatric surgery: an updated systematic review and meta-analysis, 2003-2012. JAMA Surg 2014;149:275-287.

5. Buchwald H, Oien DM. Metabolic/bariatric surgery worldwide 2011. Obes Surg 2013;23:427-436.

6. Jain D, Singhal S. Endoscopic bypass using endobarrier devices: efficacy in treating obesity and metabolic syndrome. J Clin Gastroenterol 2015;49:799-803.

7. Goyal D, Watson RR. Endoscopic bariatric therapies. Curr Gastroenterol Rep 2016;18:26.

8. Abu Dayyeh BK, Rajan E, Gostout CJ. Endoscopic sleeve gastroplasty: a potential endoscopic alternative to surgical sleeve gastrectomy for treatment of obesity. Gastrointest Endosc 2013;78:530-535.

9. Sharaiha RZ, Kedia P, Kumta N, et al. Initial experience with endoscopic sleeve gastroplasty: technical success and reproducibility in the bariatric population. Endoscopy 2015;47:164-166.

10. Sharaiha RZ, Kumta NA, Saumoy M, et al. Endoscopic sleeve gastroplasty significantly reduces body mass index and metabolic complications in obese patients. Clin Gastroenterol Hepatol 2017;15:504-510.

11. Abu Dayyeh BK, Acosta A, Camilleri M, et al. Endoscopic sleeve gastroplasty alters gastric physiology and induces loss of body weight in obese individuals. Clin Gastroenterol Hepatol 2017;15:37-43.el.

12. Lopez-Nava G, Galvão MP, da Bautista-Castaño I, Jimenez A, De Grado T, Fernandez-Corbelle JP. Endoscopic sleeve gastroplasty for the treatment of obesity. Endoscopy 2015;47:449-452.

13. Lopez-Nava G, Galvão MP, Bautista-Castaño I, Jimenez-Baños A, Fernandez-Corbelle JP. Endoscopic sleeve gastroplasty: how I do it? Obes Surg 2015;25:1534-1538.

14. Lopez-Nava G, Galvao M, Bautista-Castaño I, Fernandez-Corbelle JP, Trell M. Endoscopic sleeve gastroplasty with 1-year follow-up: factors predictive of success. Endosc Int Open 2016;4:E222-E227.

15. López-Nava Breviere G, Bautista-Castaño I, Fernández-Corbelle JP, Trell M. Endoscopic sleeve gastroplasty (the Apollo method): a new approach to obesity management. Rev Esp Enferm Dig 2016;108:201-206.

16. Galvão-Neto MD, Grecco E, Souza TF, Quadros LG, Silva LB, Campos JM. Endoscopic sleeve gastroplasty - minimally invasive therapy for primary obesity treatment. Arq Bras Cir Dig 2016;29(Suppl 1):95-97. 